\title{
Dissecting features of epigenetic variants underlying cardiometabolic risk using full- resolution epigenome profiling in regulatory elements
}

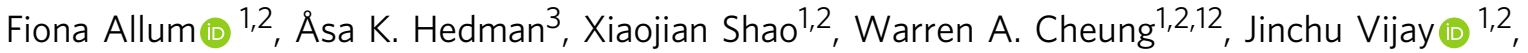 \\ Frédéric Guénard ${ }^{4}$, Tony Kwan (10) 1,2, Marie-Michelle Simon², Bing Ge ${ }^{1,2}$, Cristiano Moura ${ }^{5}$, Elodie Boulier 1,2, \\ Lars Rönnblom ${ }^{6}$, Sasha Bernatsky ${ }^{5}$, Mark Lathrop ${ }^{1,2}$, Mark I. McCarthy (1) 7,8,9, Panos Deloukas (10 10, \\ André Tchernof ${ }^{11}$, Tomi Pastinen ${ }^{1,2,12}$, Marie-Claude Vohl ${ }^{4}$ \& Elin Grundberg ${ }^{1,2,12}$
}

\begin{abstract}
Sparse profiling of $\mathrm{CpG}$ methylation in blood by microarrays has identified epigenetic links to common diseases. Here we apply methylC-capture sequencing (MCC-Seq) in a clinical population of $\sim 200$ adipose tissue and matched blood samples $\left(\mathrm{N}_{\text {total }} \sim 400\right)$, providing highresolution methylation profiling ( $>1.3 \mathrm{M} \mathrm{CpGs)}$ at regulatory elements. We link methylation to cardiometabolic risk through associations to circulating plasma lipid levels and identify lipid-associated $\mathrm{CpGs}$ with unique localization patterns in regulatory elements. We show distinct features of tissue-specific versus tissue-independent lipid-linked regulatory regions by contrasting with parallel assessments in $~ 800$ independent adipose tissue and blood samples from the general population. We follow-up on adipose-specific regulatory regions under (1) genetic and (2) epigenetic (environmental) regulation via integrational studies. Overall, the comprehensive sequencing of regulatory element methylomes reveals a rich landscape of functional variants linked genetically as well as epigenetically to plasma lipid traits.
\end{abstract}

\footnotetext{
${ }^{1}$ Department of Human Genetics, McGill University, Montréal, QC H3A OC7, Canada. ${ }^{2}$ McGill University and Genome Quebec Innovation Centre, Montréal, QC H3A 0G1, Canada. ${ }^{3}$ Department of Medicine Solna, Cardiovascular Medicine Unit, Karolinska Institute, Stockholm 171 76, Sweden. ${ }^{4}$ Institute of Nutrition and Functional Foods (INAF), Université Laval, Québec, QC GTV OA6, Canada. ${ }^{5}$ Department of Epidemiology, McGill University, Montréal, QC H3A 1A2, Canada. ${ }^{6}$ Department of Medical Sciences, Uppsala University, Uppsala 751 85, Sweden. ${ }^{7}$ Oxford Centre for Diabetes, Endocrinology and Metabolism, Churchill Hospital, University of Oxford, Old Road, Headington, Oxford OX3 7LJ, UK. ${ }^{8}$ Wellcome Centre for Human Genetics, University of Oxford, Roosevelt Drive, Oxford OX3 7BN, UK. ${ }^{9}$ Oxford NIHR Biomedical Research Centre, Oxford University Hospitals NHS Foundation Trust, John Radcliffe Hospital, Oxford OX3 9DU, UK. ${ }^{10}$ William Harvey Research Institute, Barts and The London School of Medicine and Dentistry, Queen Mary University of London, Charterhouse Square, London EC1M 6BQ, UK. ${ }^{11}$ Québec Heart and Lung Institute, Université Laval, Québec, QC G1V OA6, Canada. ${ }^{12}$ Present address: Children's Mercy Hospitals and Clinics, Kansas City, MO 64108, USA. Correspondence and requests for materials should be addressed to E.G. (email: egrundberg@cmh.edu)
} 
C omplex diseases such as obesity and type 2 diabetes (T2D) are caused by joint action of predisposing genetic and environmental factors ${ }^{1-4}$. Heritability measures of obesityrelated traits such as BMI have shown that the genetic contribution is likely only $\sim 30-40 \% 5$-pointing towards a larger impact than previously estimated by environmental effects.

CpG methylation has been shown to be disrupted in disease states $^{6,7}$ and by environmental modifiers ${ }^{8,9}$. As such, assessment of $\mathrm{CpG}$ methylation changes through epigenome-wide association studies (EWAS) enables us to connect environment and genetics $^{10,11}$ to phenotype and disease ${ }^{12}$. Circulating lipid profiles are clinically applied in cardiometabolic risk assessment ${ }^{4}$, providing indications of metabolic complications among healthy and obese individuals ${ }^{13}$. Although past EWAS efforts have successfully identified lipid-associated loci with roles in metabolic processes $^{14-18}$, we have shown the importance of using diseasetargeted tissues for functional interpretation of disease loci due to the preferential mapping of identified variants to tissue-specific regulatory elements ${ }^{11,19}$. This is an important observation considering that most EWAS to-date have studied whole-blood tissue using targeted arrays (e.g., Illumina $450 \mathrm{~K}$ array), which underrepresent distal regulatory regions (e.g., enhancers) and bias towards promoter regions. In fact, promoters are largely uninformative in EWAS due to the invariable state of resident CpGs across individuals ${ }^{11}$, partly due to insufficient sensitivity measures in DNA methylation assessments.

To overcome this limitation, we implemented the methylCcapture sequencing (MCC-seq) approach permitting simultaneous methylome and genotype profiling in regulatory regions at high resolution ${ }^{20}$. A pilot adipose tissue EWAS of triglyceride (TG) levels identified novel TG-linked methylation variation within enhancers. MCC-Seq was also applied across various tissues in hundreds of donors and demonstrated stronger enrichment of GWAS SNPs underlying allele-specific methylation within disease-linked tissues-emphasizing the importance of utilizing appropriate tissues to decipher not only epigenetic variants but genetic variants ${ }^{21}$.

Here, we present a large next-generation sequencing (NGS)based EWAS applying MCC-Seq on adipose tissue and blood samples derived from a clinically relevant cohort of obese individuals. We link $\sim 1.3 \mathrm{M}$ dynamic $\mathrm{CpGs}$ to blood plasma lipids and map positional trends of lipid-linked CpGs within functional elements. We highlight the ability of MCC-Seq to fine-map EWAS signals through replication in the large MuTHER adipose cohort and apply integrative approaches to identify diseaseassociated epigenetic variants linked to regulatory effects, further providing insight into metabolic disease etiology. We further show features of the metabolic-disease-linked methylome by assessing the contribution of genetic factors and use these tabulated associations to fine-map cardiometabolic-risk-associated GWAS SNPs.

\section{Results}

Adipose tissue epigenetic variants linked to plasma lipids. $\mathrm{CpG}$ methylation was profiled in visceral adipose tissue (VAT) from 199 severely obese individuals (BMI $>40 \mathrm{~kg} \mathrm{~m}^{-2} ; 60 \%$ female) undergoing bariatric surgery (IUCPQ, Université Laval; Supplementary Table 1; see Methods section). We applied the MCC-Seq protocol querying up to $3.3 \mathrm{M}$ CpGs mapping to adipose tissue regulatory regions ${ }^{20}$ (see Methods section). We focus on a conservative set of highly covered $(33 \times)$ and variable sites corresponding to $1.3 \mathrm{M}$ CpGs (see Methods section) that exhibited mainly (55\%) hypomethylated states $(<20 \%$ average methylation) with a smaller proportion (10\%) being hypermethylated $(>80 \%$ average methylation).
We associated $\mathrm{CpG}$ methylation at the $1.3 \mathrm{M}$ sites in adipose tissue with circulating plasma lipid levels, i.e., triglycerides (TG), HDL-cholesterol (C), LDL-C, and total cholesterol (TC) (see Methods section), applying a generalized linear model accounting for sequencing depth, age and BMI. Controlling for bias and inflation of our test-statistics was achieved using the Bayesian method $B A C O N^{22}$, noting an improvement in the inflation factor (lambda) after correction across all trait-associations (Supplementary Figures 1-4). In total, methylation levels at 1230 (FDR $10 \%$; corrected $p<3.52 \times 10^{-5}$ ) and 615 (FDR 5\%; corrected $p<$ $\left.9.25 \times 10^{-6}\right)$ CpGs were associated to at least one lipid trait (Supplementary Figure 5). We subsequently refer to "lipid-CpGs" as those reaching significant lipid associations at FDR 10\% (Supplementary Data 1). Overall, 13\% of lipid-CpGs were linked to more than one lipid trait (Supplementary Figure 5). By assessing the inter-individual variability of lipid-CpGs, these sites also depicted a more variable state than the full set of $1.3 \mathrm{M} \mathrm{CpGs}$ tested (Supplementary Figure 6).

Positioning of lipid-CpGs within regulatory elements. Identified lipid-CpGs were annotated using adipose tissue hypomethylated footprints-low-methylated regions (LMRs) and unmethylated regions (UMRs) ${ }^{20,23}$ - as indicators of regulatory elements. We previously characterized these methylated footprints $^{23}$, showing co-localization of adipose tissue LMRs and UMRs with the H3K4me1 active enhancer and H3K4me3 active promoter marks, respectively, from primary human adipocytes (NIH Roadmap Consortium). In all subsequent analyses, we refer to LMRs and UMRs as putative enhancers and promoters, respectively. We additionally characterized these adipose tissue regulatory regions in terms of their genomic lengths and discovery CpG densities, where we noted putative enhancers were shorter and less densely populated than promoters (Supplementary Table 2). Mimicking our previous findings ${ }^{20}$, lipid-CpGs were enriched in putative adipose enhancers (26\% of lipid-CpGs versus $17 \%$ in background; Fisher's exact test throughout; Fisher's $p=6.6 \times 10^{-13}$ ) while being less likely to map to putative promoters ( $40 \%$ of lipid-CpGs versus $54 \%$ in background; Fisher's $p<2.2 \times 10^{-16}$; Supplementary Figure 7 ). The set of lipid-CpGs was then restricted to include only those mapping to adipose tissue regulatory regions not shared with other tissues (i.e., wholeblood; see Methods section) and showed stronger enrichment patterns at enhancers (13\% of lipid-CpGs versus $7 \%$ in background; Fisher's $\left.p=9.9 \times 10^{-13}\right)$. Additionally, we noted a reversal of trends as lipid-CpGs were enriched in adipose-specific promoters ( $10 \%$ of lipid-CpGs versus $6 \%$ in background; Fisher's $p=8.1 \times 10^{-11}$; Supplementary Figure 7$)$. Of note, these localization patterns appear to be independent of $\mathrm{CpG}$ methylation variability at interrogated sites (Fisher's $p<1.1 \times 10^{-7}$; top 25 th percentile; Supplementary Figure 7). In total, we identified 264 putative adipose enhancers (LMRs) and 303 promoters (UMRs) harboring lipid-CpGs, of which 341 are shared elements and 226 are adipose-specific elements. These 567 regulatory elements were carried forward for further analyses (Supplementary Data 1; Fig. 1).

Given the high-density coverage of $\mathrm{CpG}$ methylation obtained through MCC-Seq, we investigated differences in positional trends of lipid-CpGs within adipose tissue hypomethylated footprints (see Methods section). Focusing first on all discovery CpGs mapping to the 264 LMRs, lipid-CpGs located more towards the mid-point of putative enhancers compared to all CpGs (Fig. 2a). CpGs locating to UMRs (within $+/-1.5 \mathrm{~Kb}$ of a transcription start site (TSS); 139/303 UMRs) exhibited a bimodal distribution flanking the TSS similar to the background with a slight peak shift downstream of the TSS further into the gene 


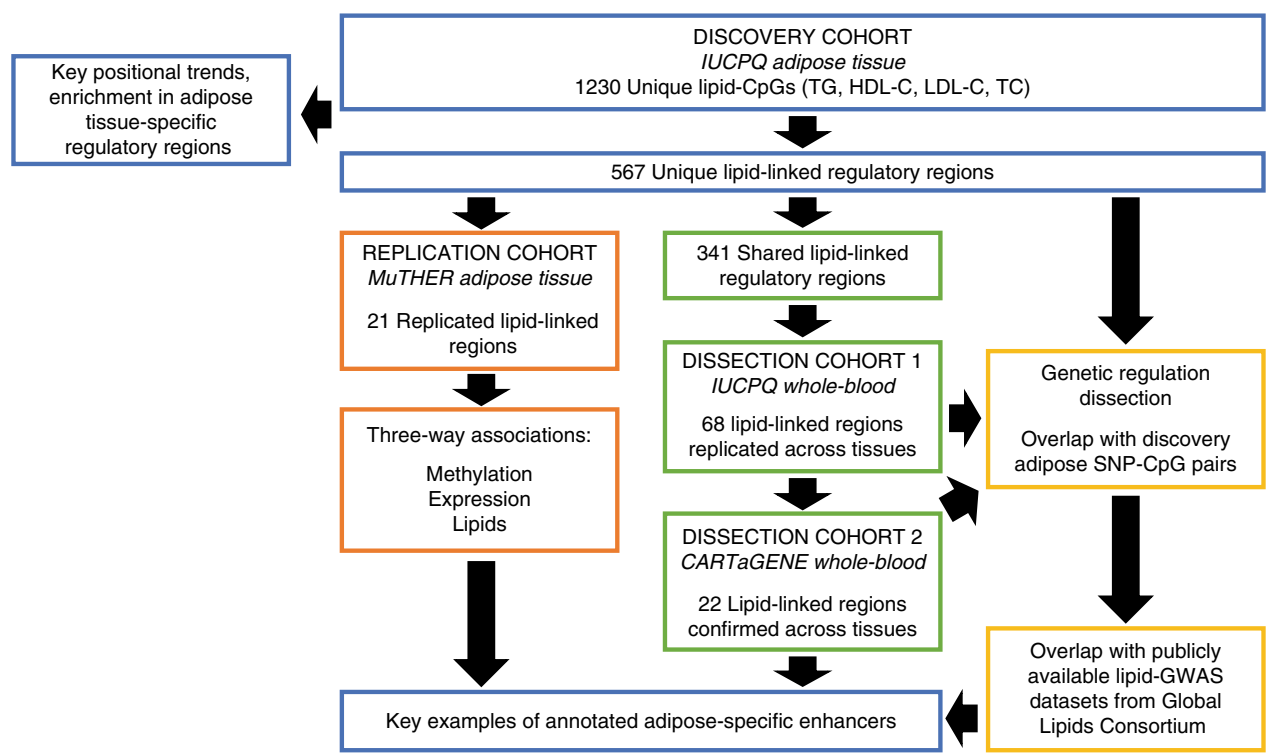

Fig. 1 Study flow chart. Overview of included study cohorts and follow-up analyses to characterize identified lipid-linked adipose tissue regulatory regions
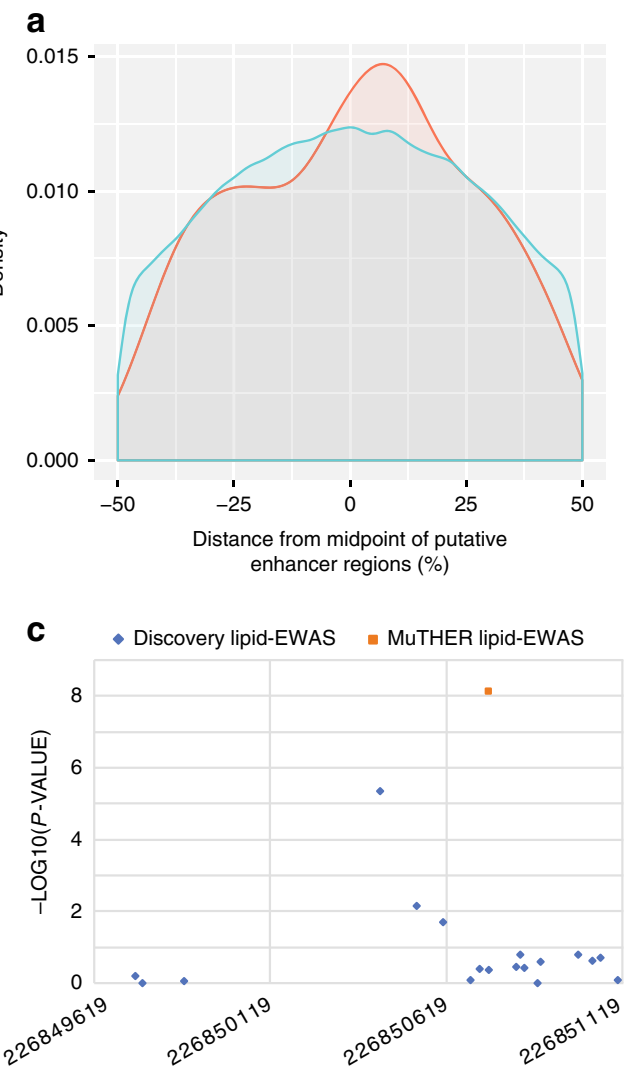

CHROMOSOME 1

\section{b}

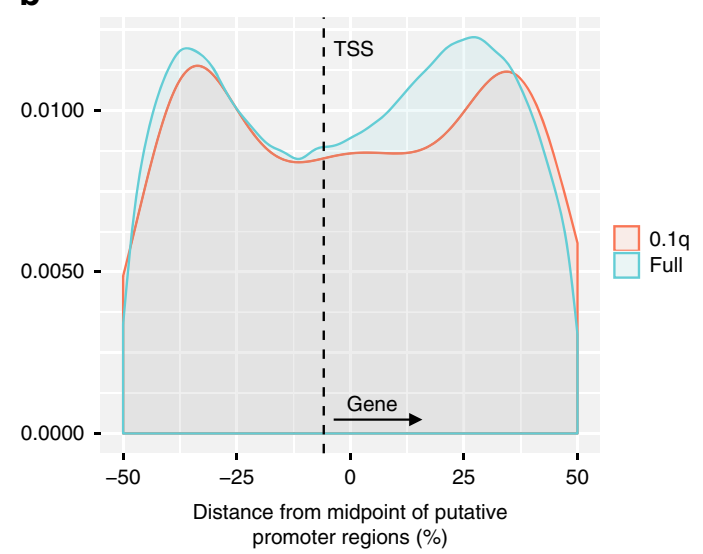

d

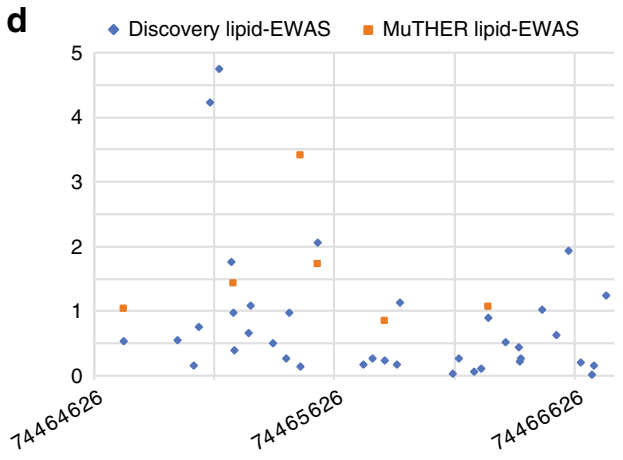

CHROMOSOME 15

Fig. 2 Positional mapping of lipid-CpGs within adipose tissue regulatory elements. Specific positional trends of significant lipid-CpGs (FDR 10\%) merged across all studied lipids traits (i.e., triglycerides, HDL-C, LDL-C, and total cholesterol) were investigated at adipose regulatory regions. Positions of CpGs were tabulated as the percent distance from the midpoint of elements (genomic distance from midpoint (bp)/length of element(bp) $\left.{ }^{\star} 100\right)$ and collapsed to summarize positional trends over all assessed elements. Positional trends are shown for a CpGs mapping to LMRs $(N=225,771)$ and $\mathbf{b}$ CpGs mapping to UMRs within $+/-1.5 \mathrm{~kb}$ of transcription start sites (TSS) not depicting bivalent gene transcription orientations (taking gene orientation into account; $N=$ 418,246). The fine-mapping potential of MCC-Seq over array-based methods is exemplified in c a replicated HDL-linked enhancer region (chr1:226849619226851122) and $\mathbf{d}$ a replicated LDL-linked promoter region (chr15:74464626-74466792), where we noted top discovery lipid-CpGs to mimic trends noted in $\mathbf{a}$ and $\mathbf{b}$ at adjacent sites to signals identified from the large-scale 450K-based MuTHER study 
body (Fig. 2b). To rule out potential technical biases explaining these observations, we assessed the mean coverage of reads within these elements and found that although lipid-CpGs have higher coverage than all assessed CpGs, the coverage does not differ based on the position of lipid-CpGs within the elements per se (Supplementary Figure 8).

Next, we contrasted the capacity of MCC-Seq to capture lipidCpGs within adipose tissue regulatory regions over alternative methods such as the Illumina $450 \mathrm{~K}^{14-18}$ and EPIC arrays. As a whole, the EPIC and $450 \mathrm{~K}$ arrays captured only 17 and $6 \%$ of the total percent CpGs profiled in LMRs by MCC-Seq and 29 and $19 \%$ of those mapping to UMRs, respectively. These percentages dropped further when focusing on CpGs typed on the arraybased methods directly overlapping MCC-Seq CpGs (Supplementary Table 3). Positional trends of CpGs in both arrays showed a depletion of coverage within putative promoters downstream of the TSS (Supplementary Figure 9)-regions towards the gene body where we showed lipid-CpGs to be enriched (Fig. 2b).

Replication of lipid-linked adipose regulatory regions. We then validated the 567 adipose regulatory regions mapping with lipidCpGs in the MuTHER cohort ( $N \sim 650$ individuals) where subcutaneous adipose tissue CpG methylation levels were profiled on the $450 \mathrm{~K}$ array ${ }^{11}$ and associated to the same lipid traits under investigation (TG, HDL-C, LDL-C, and TC) ${ }^{18}$. Of the 567 highlighted regulatory regions, only $365(64 \%)$ were covered by the $450 \mathrm{~K}$ array. In line with design biases of the $450 \mathrm{~K}$ array, a higher proportion of adipose tissue promoter regions (269/303 UMRs; 89\%) than enhancer regions (96/264 LMRs; 36\%) contained at least one $450 \mathrm{~K}$ array CpG. Using Bonferroni cutoff (taking into account each trait individually and with same direction of effect), we found the highest replication rate for TG-UMRs where $17 \%$ (13/76) of the regions were also associated with TG in the validation cohort. All replicated regions $(N=21)$ are presented in Supplementary Data 2.

To assess the potential of MCC-Seq to fine-map EWAS signals, we focused on the 16 of 21 replicated regulatory regions containing at least 2 discovery lipid-CpGs where one of these overlapped the top MuTHER lipid-CpG. Here, 15/16 (94\%) elements harbored stronger lipid associations at discovery CpGs that didn't directly overlap the top MuTHER lipid-CpG positions (Supplementary Data 2). We then investigated the localization of the "fine-mapped" discovery lipid-CpGs compared to their nearby MuTHER lipid-CpGs within the adipose tissue regulatory elements. All the "fine-mapping" discovery CpGs located at the mid-point of adipose tissue LMRs (+/ $-20 \%$ from mid-point), representing a slight increase in proportion over their paired MuTHER CpGs (2/3 CpGs). This pattern is similar to the observed positional mapping trends for the full set of lipidCpGs at LMRs, which exhibited a mid-point shift compared to all CpGs assessed (Fig. 2a, c). Likewise, "fine-mapping" discovery CpGs mapping to adipose UMRs showed that these CpGs tended to locate in greater numbers $(7 / 12 ; 58 \%)$ than their paired MuTHER CpGs (5/12 CpGs; 42\%) within the bimodal positional peaks $(+20$ to $+45 \%$ or -20 to $-45 \%$ from mid-point) previously observed for lipid-CpGs at UMRs (Fig. 2b, d). Both of these fine-mapping trends did not reach nominal significance most likely owing to the small number of observations and the additional bimodal pull of the fine-mapping exhibited at the putative promoter regions.

Functional annotation of lipid-CpGs. Replicated lipid-linked adipose hypomethylated regulatory regions were characterized by performing transcription factor binding site (TFBS) motif analyses (see Methods section). Focusing on replicated UMRs harboring lipid-CpGs ( $N=16$ regions) and excluding LMRs due to their small number $(N=5)$, TFBS linked to adipogenesis and/ or obesity related metabolic-complications were enriched, with members of the STAT family $24-26$ STAT5A ${ }^{27}$, STAT1 and STAT3 $^{28}$ being most significant, followed by NFIB ${ }^{29,30}$ and RUNX131,32 (Supplementary Table 4). We further noted that STAT5A, STAT3, and NFIB showed higher levels of expression in adipose tissues over whole-blood in the GTEx Consortium data (GTEx portal; November 2017; Supplementary Figures 10-12) with the strongest evidence for NFIB expression. We confirmed adipocyte-specific expression of NFIB through differential expression analyses of purified human adipocytes from both subcutaneous and visceral depots versus various blood cell types ( $>14.0$-fold change; $p<3.98 \times 10^{-236}$; see Methods section).

Next, replicated lipid-linked adipose tissue regulatory regions ( $N=5$ LMRs; $N=16$ UMRs) were functionally annotated by incorporating matching adipose tissue gene expression data from the MuTHER cohort ${ }^{11}$ (see Methods section). As many as 16/21 (76\%) lipid-associated regions showed significant association between the methylation status of one of their resident CpGs and the expression levels of at least one cis-located gene (FDR 10\%; range 1-9 associated genes/region; within $+/-1 \mathrm{Mb}$; Supplementary Data 3)-representing a 1.9-fold change in effect over all testable regulatory regions (10,141/26,050 regions; Fisher's $p=$ $0.00104)$. All 16 regulatory regions depicting associations to at least one gene also exhibited stronger effects on gene expression at non-adjacent genes-with an average absolute distance of $\sim 522 \mathrm{~kb}$ to their most correlated gene compared to $\sim 33 \mathrm{~kb}$ to the transcribed region of their most proximal gene. A greater proportion of these replicated lipid-associated regulatory regions (11/16 regions; $69 \%)$ correlated to the expression levels of more than one gene compared to the background (4673/10,141 regions; $46 \%$; Fisher's $p=0.08$ ).

We assessed whether the genes $(N=44)$ for which expression levels were associated with methylation status at replicated lipidlinked regions $(N=16)$ were also independently linked to the same plasma lipid phenotypes (see Methods section). As many as $77 \%$ (30/39) of testable genes linked to 15 replicated lipidassociated regulatory regions showed additional association to the same lipid trait under investigation in the expected direction of effect (Supplementary Data 4).

Restricting to genes listed in the GWAS SNP catalog $(N=20 /$ 30; accessed September 2018), we observed that $6 / 20(30 \%)$ genes associating to lipid-linked regulatory regions also showed association to metabolic-related phenotypes, revealing an enrichment of obesity-linked traits compared to the full catalog (692/ 15,815 genes; 4\%; 6.9-fold change; Fisher's $p=0.00016$; Supplementary Data 4; see Methods section). Ingenuity pathway analysis (see Methods section) of all 30 highlighted genes showed G $\alpha q$ Signaling as the most significantly associated function within this gene set $\left(p=6.94 \times 10^{-5}\right.$; Supplementary Table 5). Interestingly, two of the four genes mapped to this pathway were regulated by the same lipid-regulatory element which we follow-up in more detail below.

Tissue-specificity of lipid-linked regulatory regions. To gain insight into the potential tissue-specific nature of epigenetic signatures associated to disease, we interrogated whether lipidlinked signals mapping to regulatory regions are detectable across tissues within a study population by profiling $\mathrm{CpG}$ methylation in whole-blood from a matching set of samples $(N=206)$ from the obese IUCPQ cohort (Supplementary Table 1). We linked wholeblood methylation status to the same circulating plasma lipid levels (see Methods section) and successfully typed 565 out of the 
567 regulatory regions harboring discovery adipose tissue lipidCpGs in whole-blood, of which 340 were shared and 225 adiposespecific (i.e., not shared to whole-blood) elements (see Methods section). Globally at the same significance threshold (using Bonferroni cutoff for each trait individually and with same direction of effect), lipid-associations at shared regulatory elements replicated at a significantly higher rate (46/340 replicated lipid-linked regions; $14 \%)$ than adipose-specific elements $(12 / 225$ replicated regions; $5 \%$; Binomial test $\left.p=9.0 \times 10^{-9}\right)$. Lipidassociations at shared putative promoters (i.e., UMRs) were more likely to replicate across tissues than at shared enhancer regionswith 35/221 (16\%) lipid-linked UMRs compared to 11/119 (9\%) LMRs replicating in whole-blood. Specifically, we were able to validate associations at 4/39 (10\%) TG-LMRs, 2/39 (5\%) HDLLMRs, $7 / 34$ (21\%) LDL-LMRs, 4/26 (15\%) TC-LMRs, 10/64 (16\%) TG-UMRs, 7/69 (10\%) HDL-UMRs, 11/77 (14\%) LDLUMRs, and 14/57 (25\%) TC-UMRs in whole-blood (Supplementary Data 5). Previous studies have indicated the importance of accounting for differences in biological outcome of environmental and genetic effects on DNA methylation at the tissue level ${ }^{18}$, thus we performed the replication across adipose tissue to whole-blood by also allowing different directions of effect across tissues. Here, we were able to validate additional associations at $1 /$ 39 (3\%) HDL-LMRs, 1/34 (3\%) LDL-LMRs, 6/64 (9\%) TGUMRs, 4/69 (6\%) HDL-UMRs, 10/77 (13\%) LDL-UMRs, and 3/ 57 (5\%) TC-UMRs in whole-blood (Supplementary Data 5). Taken together, we identified 68 adipose tissue regulatory regions (13 putative enhancers and 55 promoters) showing evidence for tissue-shared lipid-associations.

Pathway analysis of the 52 genes directly overlapping the 68 tissue-independent regulatory regions (Supplementary Table 6) revealed the adipogenesis pathway as the top significantly associated function (IPA $p=3.1 \times 10^{-3}$; see Methods section). Among the genes highlighted within this pathway, we noted (1) the serine/threonine kinase AKT1 overlapping a shared promoter region (chr14:105260438-105262714) harboring CpGs positively correlated to both LDL-C and TC levels; (2) the histone deacetylase HDAC4 mapping with an intergenic enhancer region (chr2:240240338-240241584) containing CpGs depicting negative associations to HDL-C in adipose tissue that were reversed in whole-blood; (3) BMP4 overlapping a shared promoter region (chr14:54418956-54424030) where CpGs were negatively associated to TG levels. We further highlighted lipid-associated promoter regions at the following cardiometabolic risk-related loci; growth factor GDF7, kinase CERK, VGLL3 and ATP-binding cassette transporter $A B C C 5$.

Next, we investigated how the lipid-linked and tissue-shared regulatory regions identified in a clinical population associate with the same traits independently of obesity status. CpG methylation was profiled by MCC-Seq in whole-blood from a population-based $(N=137)$ cohort (CARTaGENE; https:// cartagene.qc.ca/; Supplementary Table 1), again linking wholeblood methylation status to circulating plasma lipid levels (Methods). Overall, we found 22/68 (32\%) regions to be associated with the same lipid trait under investigation in the population-based cohort (Supplementary Data 6). However, contrasting adipose lipid-associations that replicated in wholeblood with the same ( $N=46$ regions) versus opposing $(N=28$ regions) directions of effects $(N=17 / 46$ regions; $37 \%$ vs. $N=5$ / 28 regions; $18 \%$ ) showed a marked difference in the replication rate ( $>2$-fold change) indicating the possibility of the latter being more specific to the clinical condition.

Genetic contribution to lipid-CpG methylation variability. We previously validated the ability and accuracy of MCC-Seq to

\section{Table 1 Genetic regulation on lipid-linked adipose regulatory} regions

\section{Lipid-linked regulatory regions}

Adipose-specific elements $(N=226)$

Tissue-shared elements $(N=341)$

Tissue-shared elements validated in

blood cohort $1(N=68)$

Tissue-shared elements validated in

blood cohort 1 and $2(N=22)$
All lipid-linked elements $(N=567)$

Genetic regulation enrichment (fold-change)

1.5

1.1

1.7

2.1

2.2

provide genotyping information over target regions ${ }^{20}$, which we used here to study genetic effects on CpG methylation. Using this inferred genetic dataset, we integrated recently tabulated SNPCpG associations (metQTL) in cis $(+/-250 \mathrm{~kb})$ for a subset of the adipose discovery cohort ${ }^{21}$. First, we confirmed our previous findings ${ }^{11}$ that SNPs associated with $\mathrm{CpG}$ methylation are enriched in the vicinity of their linked CpGs (Supplementary Figure 13). Second, we investigated the level of genetic regulation among lipid-associated regulatory regions and noted a large fraction to be partly under genetic regulation. In line with previous studies ${ }^{11,18,33}$, we observed that $64 \%(362 / 567)$ of lipidassociated elements depicted a significant SNP-CpG association (FDR 10\%) compared to only 44\% (22101/50759) in the background (Fisher's $p<2.2 \times 10^{-16}$; Table 1). We further found that this enrichment was maintained when accounting for overall methylation variability (top $25 \%$ variable CpG methylation status across all individuals; 194/406 lipid-linked regions versus 4763/ 17593 in background; Fisher's $p<2.2 \times 10^{-16}$ ).

We queried whether the identified lipid-linked regulatory regions have different levels of genetic contribution depending on their tissue-specificity and contrasted the elements unique to adipose $(N$ $=226)$ versus those shared across tissues to whole-blood $(N=341$; Table 1). We observed an enrichment in association to cis-SNPs only at shared regulatory elements $(N=251 / 341$ regions; $74 \%$; Fisher's $p<2.2 \times 10^{-16}$; Table 1). Restricting to the subset of 68 lipid-associated shared regulatory regions that were further validated in the matched whole-blood cohort, we noted an increase in observed genetic variation contribution corresponding to as much as $93 \%\left(N=63 / 68\right.$ regions; Fisher's $p<2.2 \times 10^{-16}$; Supplementary Data 5; Table 1). Finally, we further filtered the list of lipidlinked regulatory regions to only contrast those that in addition to being validated in the matched whole-blood cohort were also significantly associated to lipids in the independent populationbased cohort (Supplementary Data 6). Here, we found a striking enrichment with 21/22 (95\%) of these tissue-independent and obese-status-independent regions to be under genetic regulation (Fisher's $p=3.3 \times 10^{-7}$ ).

To assess whether these genetically controlled lipid-linked epigenetic loci overlap GWAS loci, we incorporated GWAS SNPs for the same four lipid traits under study from the large-scale efforts of the Global Lipids Genetics Consortium ${ }^{34}$. We focused on lead SNPs associated with methylation of CpGs mapping to the 362 lipid-linked regulatory regions. Intersecting these SNPs and/or their proxies $\left(r^{2}>0.8\right)$ with the fully released dataset of GWAS SNPs at nominal significance, we noted an enrichment at lipid-linked regulatory regions for all lipid traits; TG (3.7-fold; Fisher's $p=3.4 \times$ $\left.10^{-16}\right)$, HDL-C (4.4-fold; Fisher's $\left.p<2.2 \times 10^{-16}\right)$, LDL-C (4.3-fold; Fisher's $\left.p<2.2 \times 10^{-16}\right)$ and TC (4.1-fold; Fisher's $\left.p<2.2 \times 10^{-16}\right)$. Enrichment trends were maintained at a more stringent significance cutoff for GWAS SNPs $\left(p=5.0 \times 10^{-8}\right)$ albeit with lower statistical confidence due to smaller numbers (Fisher's $p<0.05$ ). 
a

Adipose regulatory regions Whole-blood regulatory regions

b

Discovery adipose TG-EWAS

IUCPQ whole-blood TG-EWAS

CARTaGENE whole-blood TG-EWAS

Illumina 450K probes

Adipose regulatory regions

Whole-blood regulatory regions

Adipocyte H3K4me1

Peripheral blood H3K4me1

Adipocyte H3K4me3

Peripheral blood H3K4me3

C

Illumina $450 \mathrm{~K}$ probes

Illumina HT-12 probes

Adipose regulatory regions

Adipocyte H3K4me1

Adipocyte H3K4me3

d

Illumina $450 \mathrm{~K}$ probes

Illumina HT-12 probes

Adipose regulatory regions

Adipocyte H3K4me1

Adipocyte H3K4me3

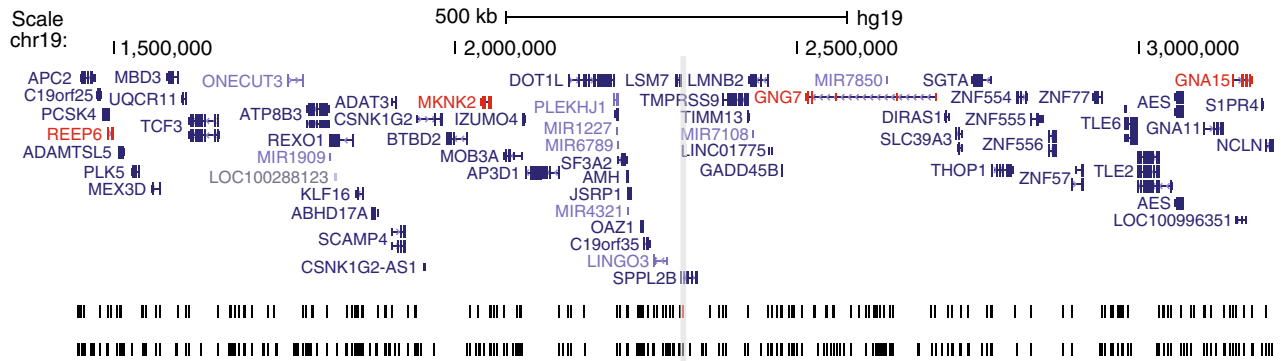

Scale
chr19:
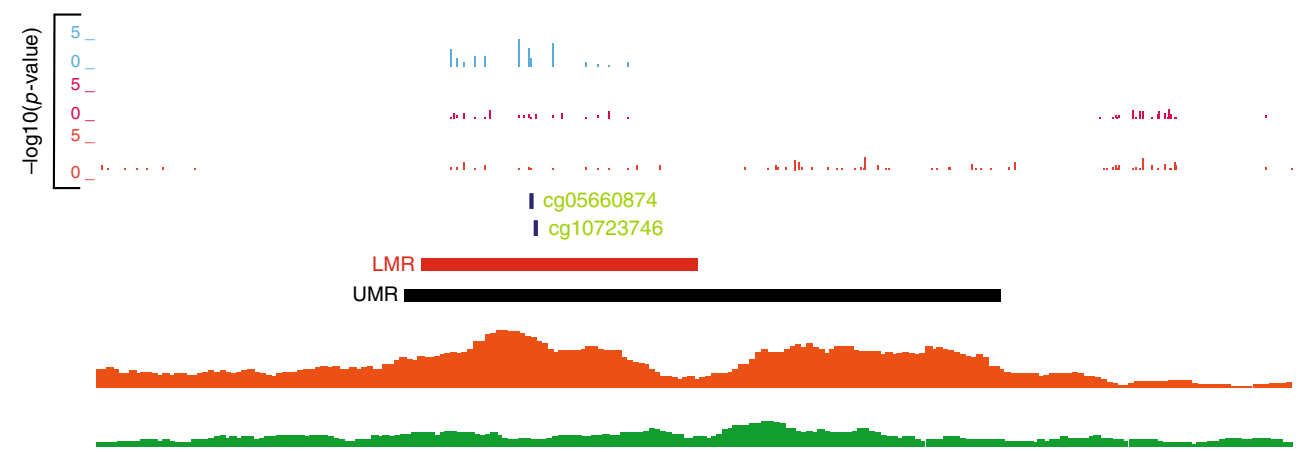

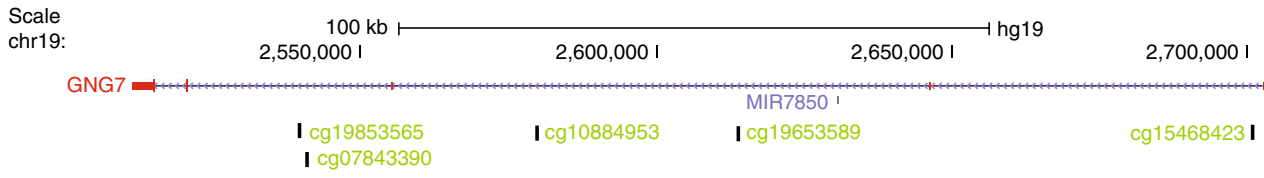
IILMN_1728107

$\begin{array}{lllll}\text { LMR I LMRI UMRI LMRI } & \text { UMR LMRI UMRI UMRI LMRI LMRI } & \text { LMRI }\end{array}$

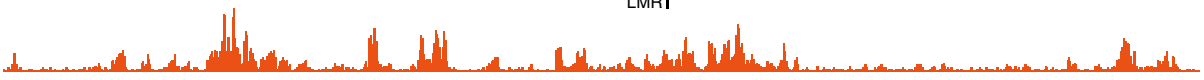

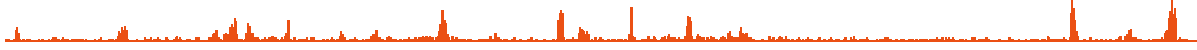

Scale $3,135,000$

$10 \mathrm{~kb}$

$3,155,000$ I

$3,165,000$ ।

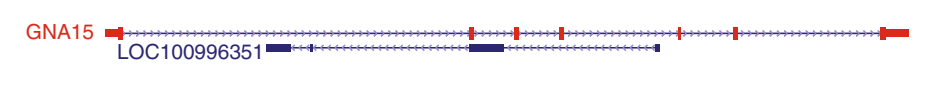

I cg26870745

cg03302088 I

ILMN_1773963 I

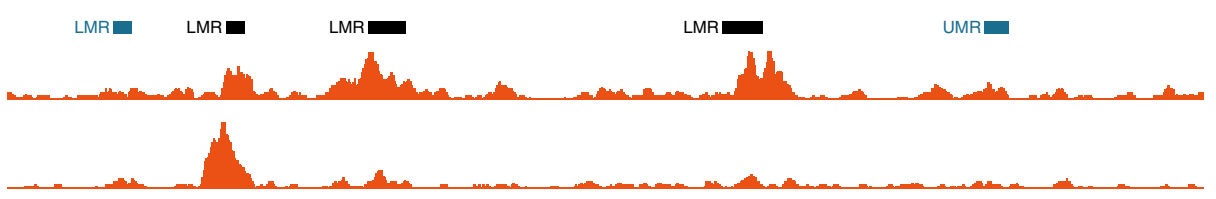

Regulation of lipid-linked adipose-specific enhancers. Genetic regulation of lipid-linked regulatory elements is pronounced among regions shared across tissue to whole-blood whereas adipose-specific regions exhibited a larger component of environmentally-driven regulation. Specifically, we found no evidence of genetic associations for 115/226 (51\%) lipid-linked regulatory regions active in adipose but not whole-blood. Among these lipid-linked regulatory regions with non-genetic regulatory effects, we followed-up on an adipose-specific putative enhancer (chr19:2332094-2333076) harboring adipose lipid-CpGs linked to TG levels in our discovery and replication samples. (Supplementary Data 2). This enhancer region maps to the first intragenic region of $S P P L 2 B$ - a locus with no reported associations to cardiometabolic risk (Fig. 3a, b). We initially highlighted the 
Fig. 3 TG-linked adipose-specific regulatory region shows putative pleiotropic effects. A top discovery TG-CpG (chr19:2332436; corrected $p=2.4 \times 10^{-5}$; sky blue track) replicated by multiple nearby MuTHER TG-CpGs (cg05660874; $p=5.1 \times 10^{-10}$; cg10723746; $p=1.0 \times 10^{-8}$; light green track) locates within an adipose-specific enhancer region (chr19:2332094-2333076) overlapping the first intron of SPPL2B (LMR; shown in red in a the broad and b zoomed-in view). Methylation levels at cg05660874 and cg10723746 show associations to cis-locating REEP6, MKNK2, GNG7, and GNA15 (highlighted in red in a), which in turn exhibit associations to TG levels in the MuTHER cohort with GNG7 and GNA15 showing the strongest links (c GNG7; cg05660874 versus ILMN_1709247; $p=4.9 \times 10^{-5}$; cg10723746 versus ILMN_1709247; $p=3.1 \times 10^{-8}$; ILMN_1709247 versus TG; $p=1.2 \times 10^{-12}$; d GNA15; ILMN_1773963 versus cg10723746; $p=1.5 \times 10^{-17}$; ILMN_1773963 versus cg05660874; $p=1.5 \times 10^{-16}$; ILMN_1773963 versus TG; $p=1.5 \times 10^{-18}$ ). We show evidence for a co-regulation network between these two genes and the enhancer region by highlighting associations between $450 \mathrm{~K}$ array probes (light green tracks in b-d) locating to several regulatory regions (shown in red in $\mathbf{b}$ and teal in c, d) and expression levels of $\mathbf{c}$ GNG7 and d GNA15 in MUTHER. We show a lack of whole-blood lipid-EWAS signals at the enhancer of interest (b), which is supported by the adipocyte-specific nature of chromatin signatures observed at the locus (Roadmap Epigenomics Consortium; adipocyte nuclei donor 92 shown in orange versus peripheral blood donor TC015 shown in green)

region for harboring TG-linked methylation in the discovery cohort near the mid-point of the enhancer region (chr19:2332436; corrected $p=2.4 \times 10^{-5}$; Fig. $3 \mathrm{~b}$ ) - mimicking positional trends for lipid-CpGs at this type of element. The positive correlation of methylation to TG levels at this region was validated in the largepopulation based MuTHER cohort at nearby CpGs (cg05660874; $p=5.1 \times 10^{-10} ; \quad$ cg10723746; $\quad p=1.0 \times 10^{-8} ; \quad$ Supplementary Data 2; Fig. 3b). Confirming earlier results for the characterization of adipose putative enhancers ${ }^{23}$, overlapping the intragenic region with adipocyte-specific H3K4me1 and H3K4me3 (Roadmap; donor 92) showed co-localization of the highlighted adipose-specific LMR with the H3K4me1 enhancer mark (Fig. 3b). This was not observed in peripheral blood (Roadmap; donor TC015) ChIP-Seq data as H3K4mel peaks were absent, indicating the adipose-specific nature of the regulatory marks (Fig. 3b). This observation corroborates the lack of replication of epigenetic regulation from whole-blood EWAS at this element (Supplementary Data 5; Fig. 3b).

Integrating the MuTHER cohort expression data (see Methods section) revealed a lack of significant epigeneticassociation to expression levels of the SPPL $2 B$ locus. In line with the trend reported above, we instead noted that expression levels of GNA15-located $803 \mathrm{~kb}$ downstream of the putative enhancer region-were the most correlated (ILMN_1773963 versus cg10723746; $p=1.5 \times 10^{-17}$; ILMN_1773963 versus $\operatorname{cg} 05660874 ; p=1.5 \times 10^{-16}$; Fig. 3d). We further observed links to expression levels of GNG7 (179 kb downstream), REEP6 (834 kb upstream) and MKNK2 (281 kb upstream; Supplementary Data 3; Fig. 3a). Expression levels of these four genes were also associated with TG levels in the MuTHER cohort, with GNA15 and GNG7 exhibiting the strongest relationships (GNA15; ILMN_1773963; $\quad p=1.5 \times 10^{-18} ; \quad$ GNG7; ILMN_1728107; $p=1.2 \times 10^{-12}$; Supplementary Data 4 ; Fig. $3 \mathrm{c}$, d) - corroborating the link between regulation of these loci with levels of TG and disease state. Supporting a co-regulation network between these genes is the strong correlation between the $450 \mathrm{~K}$ array probes located at several regulatory regions at these genes and the expression products of GNG7 and GNA15 interchangeably (Supplementary Data 7; Fig. 3c, d). GNA15 and GNG7 both encode for G-protein subunits with suggested roles for GNA15 in heart failure ${ }^{35}$ and glucose homeostasis ${ }^{36}$ and for GNG7 in coronary artery calcification ${ }^{37}$ (Supplementary Data 4). Both of these genes mapped to the top IPA diseaselinked function of $G \alpha q$ Signaling for genes under regulation by replicated lipid-linked regulatory regions (IPA $p=6.94 \times 10^{-5}$; Supplementary Table 5). Taken together, this may suggest that the identified adipose-specific regulatory region has pleiotropic effects regulating both GNA15 and GNG7 expression, resulting in additive disease risk.

Although we observed a lack of enrichment for genetic associations among lipid-linked regulatory regions active in adipose but not whole-blood, we identified 111 regions under genetic regulation. To exemplify this, we focused on an element mapping to an intragenic region of GALNT2 (chr1:230312462230313455) showing both epigenetic and genetic associations to HDL-C (Fig. 4; Supplementary Data 1). Specifically, we showed that this lipid-linked regulatory region (corrected $p=2.0 \times 10^{-5}$ ) is under tight genetic regulation with seven CpGs associating to multiple SNPs $(N=21)$ flanking this element (Supplementary Data 8; Fig. 4b). These lead SNPs were in high LD $\left(r^{2}>0.9\right)$ with an HDL-linked GWAS SNP ${ }^{34}$ (Global Lipids Consortium; rs627702; $p=5.0 \times 10^{-24}$ ) located $11 \mathrm{~kb}$ downstream of the enhancer (Fig. 4). Of note, this HDL-linked GWAS SNP was independent of the top GWAS SNP reported by the Global Lipids Consortium study for this same trait, which locates upstream of the enhancer region (rs4846914; $p=4.0 \times 10^{-41}$; Fig. $\left.4 \mathrm{a}\right)^{34}$. Genetic effects at this enhancer were supported by conditional analysis where absence of lipid-CpG association was noted when genotypes were included in the model with rs2760537 being the most prominent (corrected $p=4.3 \times 10^{-2} ; q=0.78$; see Methods section). Dissecting results with whole-blood EWAS showed the adipose-specific nature of HDL-association at this region (Fig. 4). This enhancer is also not covered on the $450 \mathrm{~K}$ array, representing a novel avenue for HDL-association to epigenetic variants. In addition, we found no evidence of cis-eQTLs (GTEx Consortium) linking genetic variants at this locus to gene expression (Fig. 4b). This observation in combination with the lack of a strong adipocyte-specific H3K27ac signature at this enhancer indicates a possible poised or primed region state, supporting efforts highlighting the superior molecular value provided by epigenetics traits over gene expression alone ${ }^{38}$. The glycosyltransferase GALNT2 locus itself has previously been associated to metabolic syndrome ${ }^{39}$, TG levels ${ }^{40,41}$ and type 2 diabetes $^{42}$, with our current results supporting additional links to cardiometabolic disease through putative epigenetic regulation.

\section{Discussion}

We recently introduced MCC-Seq ${ }^{20}$ as a cost-effective and flexible platform for simultaneous DNA methylation and genotype interrogation in large-scale cohorts, permitting targeted and dense profiling of active methylomes within disease-relevant tissues. Here, we apply MCC-Seq in a comprehensive epigenomewide study of plasma blood lipids (including TG, HDL-C, LDL-C, and TC) and identify 567 lipid-linked regulatory regions in visceral adipose tissue. We combine a stringent statistical correction method (BACON) with a more lenient FDR threshold (10\%) and perform detailed follow-ups on regions replicating across adipose tissue depots and across tissue types (whole blood) using the classical Bonferroni approach. This strategy allowed us to present an expanded resource of cardiometabolic risk-linked epigenetic loci. 
a

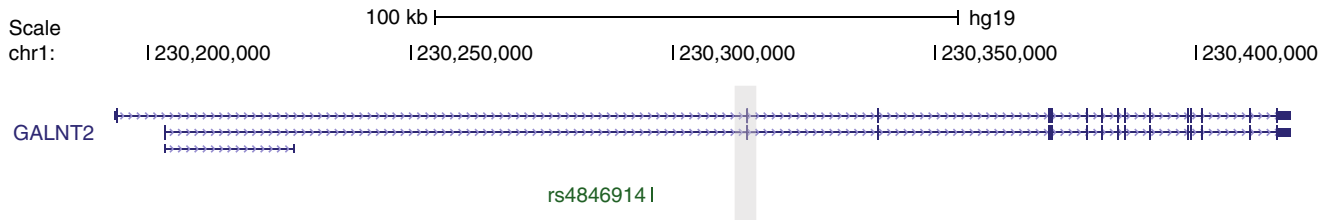

NHGRI-EBI catalog of GWAS

Top independent HDL-GWAS SNP

Irs627702

Discovery adipose HDL-EWAS

IUCPQ whole-blood HDL-EWAS

CARTaGENE whole-blood HDL-EWAS

Adipose regulatory regions

Whole-blood regulatory regions

Adipose WGBS profile

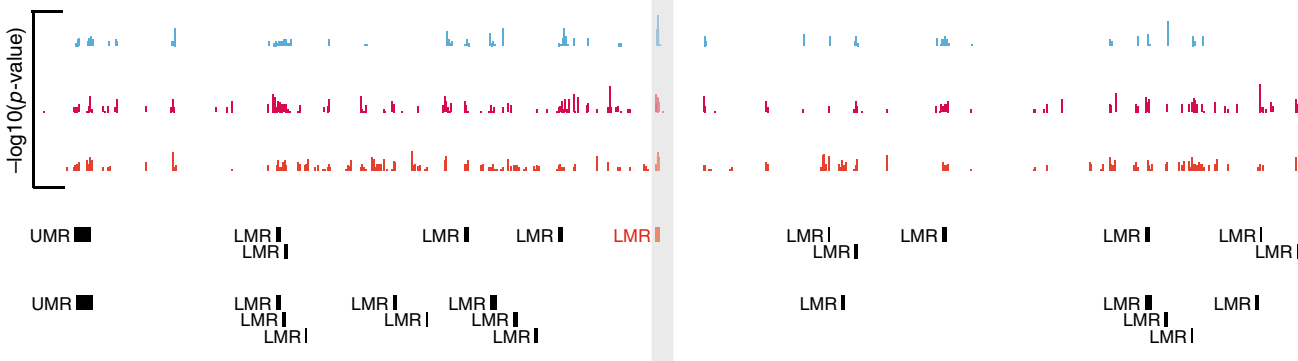

Adipocyte RNA-Seq (forward)

Peripheral blood RNA-Seq (merged)

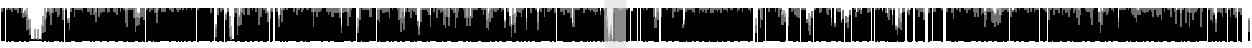

b

b

Scale
chr1:

$5 \mathrm{~kb} \longmapsto \mathrm{hg} 19$

GALNT2

1230,310,000

| 230,315,000

| 230,320,000

$1230,325,000$

Top independent HDL-GWAS SNP

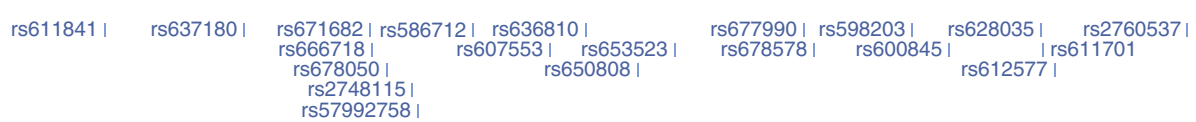

Top metQTL SNPS

Discovery adipose HDL-EWAS

IUCPQ whole-blood HDL-EWAS

CARTaGENE whole-blood HDL-EWAS

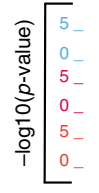

rs57992758

Illumina $450 \mathrm{~K}$ probes

Adipose regulatory regions

Adipocyte H3K4me1

Peripheral blood H3K4me1

Adipocyte H3K27ac

Peripheral blood H3K27ac

Adipocyte H3K4me3

Peripheral blood H3K4me3

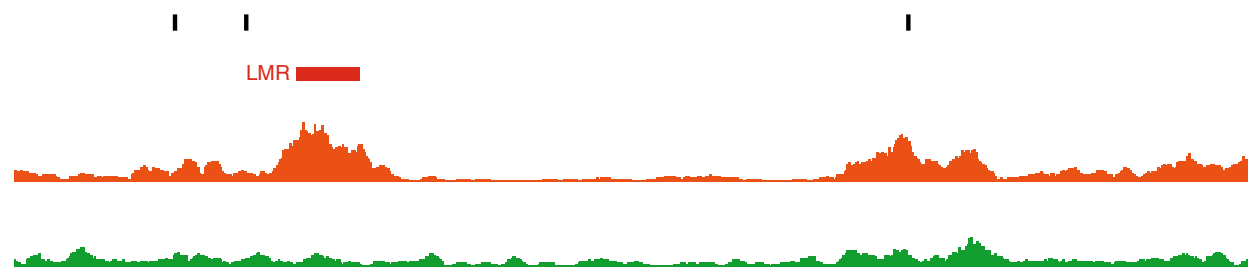

I..1

1..山 山 ।

.... لا

l...

I

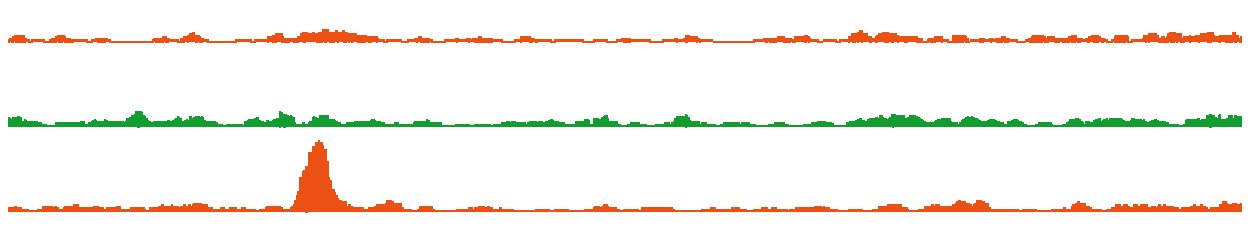

We confirm current epigenomics trends where tissue-specific regulatory regions such as enhancers globally appear more likely to contain trait-linked CpGs compared to promoters - emphasizing the importance of targeting these regions to expand our understanding of complex disease biology. The observed underrepresentation of lipid-associated epigenetic variants within promoters may be attributable to the more tightly regulated and static nature of these elements, where smaller variations may be biologically impactful but harder to statistically identify. Using MCC-Seq for dense single-base resolution profiling at regulatory elements (7 CpGs/LMR and $37 \mathrm{CpG/UMR,} \mathrm{respectively)} \mathrm{is}$ advantageous by depicting unique positional trends of lipid- 
Fig. 4 HDL-C linked adipose-specific regulatory region under genetic regulation. A discovery HDL-CpG (chr1:230313001; corrected p=2.0 $\times 10^{-5}$; sky blue track) maps within an intragenic region of GALNT2 (chr1:230312462-230313455) overlapping an adipose-specific putative enhancer region (LMR; shown in red in $\mathbf{a}$ the broad and $\mathbf{b}$ zoomed-in view). The adipose-specific nature of the epigenetic signature at this locus is supported by patterns in adipocyte nuclei (Roadmap Epigenomics Consortium; donor 92 for H3K4me1 and H3K4me3; donor 7 for H3K27ac; orange tracks) versus peripheral blood (Roadmap Epigenomics Consortium; donor TC015; green tracks) chromatin marks as well as from intersecting whole-blood EWAS signals (pink and dark orange tracks). We show that the enhancer region is under extensive genetic regulation by nearby cis-SNPs (gray blue tracks in $\mathbf{b}$ ) that are in high LD ( $r^{2}>0.9$ ) with an HDL-linked GWAS SNP (Global Lipids Consortium; rs627702; $p=5.0 \times 10^{-24}$; purple tracks), which is independent of the previously reported top $\mathrm{HDL}$-linked SNP at this locus ( $r 54846914 ; p=4.0 \times 10^{-41}$; dark green track in a). We depict a lack of coverage of the $450 \mathrm{~K}$ array at this region. Adipocytespecific (in-house data; light orange track) and peripheral blood RNA-Seq (Roadmap Epigenomics Consortium; donor TC014; light green track) data at the locus is also depicted in a

associated epigenetic variants. Key differences are observed in positioning at putative enhancers in contrast to promoters: lipidassociated epigenetic variants show clear enrichment at the midpoint of enhancers whereas they depict a bimodal distribution flanking the TSS of promoters. These observations may reflect the TFBS landscape within regulatory regions with preferential binding of TFs at midpoints or edges dependent on elements. Comparisons of these full-resolution positional trends with those captured by array-based approaches exemplified the limitations of the latter methods to assess CpGs within regulatory regions both in terms of the number of $\mathrm{CpG}$ s covered and ascertainment biases due to probe design.

We further demonstrate that NGS-based high resolution CpG profiling in epigenome-wide studies allows for fine-mapping of trait-linked epigenetic signals from large-scale array-based studies. Due to limitations in visceral adipose tissue cohort availabilities, the large MuTHER subcutaneous adipose tissue cohort was used as the best alternative proxy of available data for replication studies, therefore we focused on epigenetic variants stable across these two adipose tissue depots. We highlight a highconfidence set of 21 adipose-specific regulatory regions associated with plasma lipid levels. Identified signals for $>90 \%$ of lipidassociated regulatory regions were refined, with "fine-mapping" discovery CpGs mimicking positional trends highlighted at adipose regulatory elements. We hypothesize that differences in study design both in terms of adipose tissue depots (visceral versus subcutaneous) and cohort selection (obese versus population-based) between the discovery study and MuTHER cohorts, respectively, may contribute to the observed replication rate. Nevertheless, our TFBS analysis provided insight into potential underlying signaling pathways. Specifically, binding motifs for $N F I B$ were enriched at adipose tissue promoter regions mapping with lipid-associated epigenetic variants. Interestingly, $N F I B$ has previously been reported to function in glucose transport ${ }^{29}$ and also serves as an important regulator of proper adipocyte differentiation as exemplified by preferential mapping to adipocytespecific or preadipocyte-specific open chromatin peaks ${ }^{29,30}$.

While MCC-Seq represents added fine-mapping value for fullresolution methylome assessment, past profiling efforts within the MuTHER cohort have provided us with rich array-based datasets. Linking methylation, expression, and phenotype profiles across $\sim 600$ adipose tissue samples, we identify lipid-linked replicated adipose tissue regulatory regions associating to plasma lipid traits and expression levels at unique loci that associate to the same lipid traits. We highlight several obesity-related GWAS loci$C S K^{43}, S L C O 3 A 1^{44}$ and $G N G 7^{37}$ and GNA15 35,36 - and report several novel genes including LCN2, ECHS1, IDH2, and CD7. These genes also map to metabolic disease-linked pathways such as the highlighted G $\alpha q$ Signaling known to have a role in adipogenesis through its action in regulating intracellular calcium levels and downstream expression of the master regulators PPAR $\gamma$ and $\mathrm{C} / \mathrm{EBPa}^{45,46}$.
CpG methylation is seen as a proxy linking genetics and environment to disease and phenotype. To further contribute to our understanding of genetic and non-genetic factors impacting complex diseases, we dissect lipid-linked regulatory regions through adipose SNP-CpG associations ${ }^{21}$ within the same cohort. As previously observed ${ }^{11,18,33}$, a large fraction of lipid-associated regulatory elements is under genetic regulation. These genetic effects are strengthened when restricting to tissue-independent and lipid-linked regions replicating to whole-blood within the same disease-cohort (93\%) as well as across cohorts (95\%)hinting at a coordinated mechanistic regulation over these regions across tissues. We highlight an adipose tissue specific putative enhancer on chromosome 1 -locating within the first intron of the obesity-linked GALNT2 $2^{39-42}$, where methylation levels at this regulatory region are under genetic control by variants within an HDL-linked GWAS locus ${ }^{34}$. Adipocyte-specific histone marks at the locus suggest that the HDL-linked regulatory region represents an adipose-specific poised enhancer and may explain why genetic regulation of this disease locus has not been identified by large eQTL efforts such as from the GTEx Consortium. This finding highlights the importance of studying epigenetic marks such as DNA methylation over gene expression alone.

Building on a previous study ${ }^{11}$, we also present an expanded methylation-expression association analysis, permitting us to assess pleiotropic effects of adipose tissue regulatory regions showing association to cardiometabolic risk factors. In line with current chromatin conformational studies, we report that methylation status at lipid-linked regulatory regions shows stronger associations to expression levels of genes locating $~ 500$ $\mathrm{kb}$ away, on average. A majority $(\sim 70 \%)$ of these lipid-linked regulatory elements exhibit putative pleiotropic effects-indicating the occurrence of regulatory networks linked to the disease state. We focus on an adipose tissue-specific TG-linked enhancer region on chromosome 19 showing strong putative effects on the expression levels of two $G \alpha q$ Signaling genes-glucose homeostasis-linked GNA $15^{36}$ and coronary artery disease-linked $G N G 7^{37}$ located $>200 \mathrm{~kb}$ upstream of the element. We support observed TG-associations at this region through our three-way associations of methylation, expression and lipids within the MuTHER cohort. We also present evidence for a co-regulation network between regulatory regions mapping to GNA15 and GNG7 and the adipose enhancer of interest. Taken together, this may suggest that the identified adipose-specific regulatory region has pleiotropic effects regulating expression of both GNA15 and GNG7 resulting in additive disease risk.

In conclusion, our study demonstrates the advantage of NGSbased methylome profiling in disease-relevant tissues to identify complex trait-linked epigenetic variants at high resolution. We show that targeted sequencing approaches enables us to refine methylome landscape features and to further disentangle the genetic versus environmental contributions to complex traits. Our study represents an expanded dataset of cardiometabolic-risk- 
linked epigenetic regulatory regions in the disease-relevant adipose tissue. Our findings confirm that integrating cellular phenotypes with disease traits across tissues enables the identification of functional epigenetic variants in regulatory regions linked to complex disease traits.

\section{Methods}

Sample collections. We obtained 199 visceral adipose tissue (VAT) samples (males $N=79$; females $N=120$ ) from the Quebec Heart and Lung Institute for our discovery cohort (IUCPQ; Université Laval, Quebec City, Canada). Samples were collected between June 2000 and July 2012 for 1906 severely obese (BMI $\left.>40 \mathrm{~kg} \mathrm{~m}^{-2}\right)$ men $(N=597)$ and women $(N=1309)$ undergoing biliopancreatic diversion with duodenal switch ${ }^{47}$ at this Institute as previously described ${ }^{48}$. Briefly, subjects fasted overnight before the surgical procedure. Anesthesia was induced by a short-acting barbiturate and maintained by fentanyl and a mixture of oxygen and nitrous oxide. VAT samples were obtained within $30 \mathrm{~min}$ of the beginning of the surgery from the greater omentum ${ }^{48}$.

We additionally obtained 206 whole-blood samples from the same IUCPQ cohort described above for dissection of adipose epigenetic variants. Blood was collected before surgery.

The sample collection was approved by the Université Laval and McGill University (IRB FWA00004545) ethics committee and performed in accordance with the principles of the Declaration of Helsinki. Tissue banking and the severely obese cohort were approved by the research ethics committees of the Quebec Heart and Lung Institute. All participants provided written informed consent before enrollment in the study.

We included 137 whole-blood samples from the CARTaGENE cohort (https:// cartagene.qc.ca/) in the study design for dissection of adipose epigenetic variants. As a whole, the CARTaGENE cohort numbers $\sim 20,000$ general population subjects drawn from the province of Québec, Canada. Using bio-banked serum from a random subset $(N=3600)$ of the CARTaGENE cohort, ACPA (anti-citrullinated protein antibody) positive subjects $(N=69 ; 18$ with high titers $\geq 60$ units, the others with medium titers $=20-59$ units) were identified by an enzyme-linked immunosorbent assay (Quanta Lyte, CCP3 IgG: Inova Diagnostics Inc., San Diego, CA). Age and sex-matched ACPA negative subjects $(N=68)$ were randomly selected. ACPA status was not considered as a covariate in this study.

The methylation studies of the samples from CARTaGENE were approved by the McGill University institutional review board, IRB number A04-M46-12B. All participants provided written informed consent before enrollment in the study.

BMI was calculated as weight in kilograms divided by height in meters squared. Plasma total cholesterol (TC), triglyceride (TG), and high-density lipoprotein cholesterol (HDL-C) levels were measured using enzymatic assays. HDL-C was measured in the supernatant following precipitation of very low-density lipoproteins and low-density lipoproteins with dextran sulfate and magnesium chloride. Plasma low-density lipoprotein cholesterol (LDL-C) levels were estimated with the Friedewald formula. Summary of the characteristics are tabulated in Supplementary Table 1 .

MCC-Seq methylation profiling. Genomic DNA was extracted from the blood buffy coat using the GenElute Blood Genomic DNA kit (Sigma, St. Louis, MO, USA) and quantified using both NanoDrop Spectrophotometer (Thermo Scientific) and PicoGreen DNA methods. The samples were profiled through targeted methylation sequencing as previously described ${ }^{20}$. Briefly, in MCC-Seq a wholegenome sequencing library is prepared and bisulfite converted, amplified and a capture enriching for targeted bisulfite-converted DNA fragments is carried out. The captured DNA is further amplified and sequenced. More specifically, wholegenome sequencing libraries were generated from 700 to $1000 \mathrm{ng}$ of genomic DNA spiked with $0.1 \%$ (w/w) unmethylated $\lambda$ DNA (Promega) previously fragmented to 300-400 bp peak sizes using the Covaris focused-ultrasonicator E210. Fragment size was controlled on a Bioanalyzer DNA 1000 Chip (Agilent) and the KAPA High Throughput Library Preparation Kit (KAPA Biosystems) was applied. End repair of the generated dsDNA with $3^{\prime}$-overhangs or $5^{\prime}$-overhangs, adenylation of $3^{\prime}$-ends, adapter ligation and clean-up steps were carried out as per KAPA Biosystems' recommendations. The cleaned-up ligation product was then analysed on a Bioanalyzer High Sensitivity DNA Chip (Agilent) and quantified by PicoGreen (Life Technologies). Samples were then bisulfite converted using the Epitect Fast DNA Bisulfite Kit (Qiagen), according to the manufacturer's protocol. Bisulfiteconverted DNA was quantified using OliGreen (Life Technologies) and, based on quantity, amplified by 9-12 cycles of PCR using the Kapa Hifi Uracil + DNA polymerase (KAPA Biosystems), according to the manufacturer's protocol. The amplified libraries were purified using Ampure Beads and validated on Bioanalyzer High Sensitivity DNA Chips, and quantified by PicoGreen. SeqCap Epi Enrichment System protocols (Roche NimbleGen) were then carried out for the capture step using the previously presented adipose-specific custom panels ${ }^{20} \operatorname{MetV} 1(N=113$ discovery adipose samples), MetV2 $(N=92$ discovery adipose samples; $N=206$ whole-blood IUCPQ cohort samples) as well as a whole-blood-specific custom panel $^{49}$ ( $N=137$ CARTaGENE cohort samples). The hybridization procedure of the amplified bisulfite-converted library was performed as described by the manufacturer, using $1 \mu \mathrm{g}$ of total input of library, which was evenly divided by the libraries to be multiplexed, and incubated at $47^{\circ} \mathrm{C}$ for $72 \mathrm{~h}$. Washing and recovering of the captured library, as well as PCR amplification and final purification, were carried out as recommended by the manufacturer. Quality, concentration and size distribution of the captured library was determined by Bioanalyzer High Sensitivity DNA Chips. Captures were sequenced on the Illumina HiSeq2000/ 2500 system using 100-bp paired-end sequencing.

Reads were aligned to the bisulfite converted reference genome using BWA v.0.6.1 $1^{50}$. We removed (i) clonal reads, (ii) reads with low mapping quality score $(<20)$, (iii) reads with more than $2 \%$ mismatch to converted reference over the alignment length, (iv) reads mapping on the forward and reverse strand of the bisulfite converted genome, (v) read pairs not mapped at the expected distance based on library insert size, and (vi) read pairs that mapped in the wrong direction as described by Johnson et al. ${ }^{51}$ To avoid potential biases in downstream analyses, we applied our benchmark filtering criteria as follows; $\geq 5$ total reads, no overlap with SNPs (dbSNP 137), $\leq 20 \%$ methylation difference between strands, no offtarget reads and no overlap with DAC Blacklisted Regions (DBRs) or Duke Excluded Regions (DERs) generated by the ENCODE project: (http://hgwdev.cse. ucsc.edu/cgi-bin/hgFileUi?db=hg19\&g=wgEncodeMapability).

Methylation values at each site were calculated as total (forward and reverse) non-converted C-reads over total (forward and reverse) reads. CpGs were counted once per location combining both strands together. We restricted the analyses to CpGs covered in at least 100 individuals for the IUCPQ cohorts and 50 individuals for the CARTaGENE cohort (due to the smaller cohort size) with more than $10 \%$ of these having methylation status above zero and below $100 \%$.

Epigenome-wide association of plasma lipid levels. We tested associations between methylation levels of CpGs detected by MCC-Seq with circulating lipid levels (TG, HDL-C, LDL-C, and TC) from the corresponding cohorts using a generalized linear model (GLM) function implemented in R3.1.1. Outliers in lipid levels were identified by setting a cutoff of mean $\pm 3 *$ SD and removed from further analysis. Lipid levels not depicting a normal distribution were converted to the log scale (adipose IUCPQ: TG; whole-blood IUCPQ: TG and HDL-C; CARTaGENE: TG, HDL-C, and LDL-C). The response variable (methylation levels) was fitted to a binomial distribution weighted for sequence read coverage at each site and adjusted (1) for age, sex, MCC-Seq panel batch effect and BMI for discovery cohort adipose samples, and (2) for age, sex, blood cell proportions and BMI for the whole-blood IUCPQ and CARTaGENE cohort samples. We remove bias and inflation by applying the bacon correction ${ }^{22}$ on the test statistics using default parameters. False-discovery rate (FDR) was calculated with the R/Bioconductor $q$ value package $^{52}$ for each trait individually in the adipose IUCPQ cohort. We set the significance level at FDR 10\%. Bonferroni cutoff was used as a significance threshold for dissection with the whole-blood cohorts for each trait individually.

Subcutaneous adipose tissue methylation data from a population-based cohor of 648 female individuals in the TwinsUK/MuTHER cohort was obtained for replication. The samples were profiled on the Illumina $450 \mathrm{~K}$ array and normalized as described previously ${ }^{11}$. Associations between $450 \mathrm{~K}$ array methylation data $(N=$ 355,296 CpG probes) and the four circulating lipid levels under investigation were previously assessed ${ }^{18}$ using a linear mixed model taking into account familial relationship, twin zygosity and other cofactors into account (i.e., age, beadchip, BS conversion efficiency, BS-treated DNA input and BMI-expect when assessing BMI itself). Bonferroni cutoff was used as a significance threshold for validation for each trait individually.

Positional mapping analyses. We defined un-methylated (UMR) and lowmethylated regions (LMR) by mining through whole-genome bisulfite sequencing datasets from adipose and whole-blood samples from the same cohort, separately, as described previously ${ }^{20,23}$. Through these efforts, we reported 20,195 UMRs and 45,065 LMRs for adipose tissue and 19,871 UMRs and 46,159 LMRs for wholeblood samples 20,23 . Adipose-specific regions were previously defined by intersecting adipose and whole-blood hypomethylated regions, where 2342 and 24,687 adipose-specific UMRs and LMRs were tabulated, respectively ${ }^{20}$.

Positional trends of $\mathrm{CpGs}$ within adipose regulatory elements were assessed restricting to LMRs containing at least $1 \mathrm{CpG}(N=31,964)$ and UMRs containing at least $1 \mathrm{CpG}$ and within $+/-1.5 \mathrm{~kb}$ of transcription start sites (TSS) as well as not depicting bivalent gene transcription orientations $(N=10,924)$. Position of CpGs were tabulated as the percent distance from the midpoint of elements (genomic distance from midpoint (bp)/length of element $\left.(b p)^{\star} 100\right)$ and collapsed to make density plots using ggplot $2^{53}$ to summarize positional trends over all assessed elements. Gene orientation was additionally taken into account for CpGs mapping to UMRs where UMRs were positioned upstream of genes.

Transcription factor binding site motif analysis. Transcription factor binding site (TFBS) motif analysis was performed using the Homer software ${ }^{54}$ for lipidlinked UMRs ( $N=16$ regions) replicated in the MuTHER cohort where we excluded replicated lipid-linked LMRs due to their small number ( $N=5$ regions) Default settings were selected with the "given" size option. UMRs harboring replicated lipid-associated CpGs were contrasted against the remaining promoter regions containing interrogated $\mathrm{CpGs}$ that lacked nominal significance in the 
discovery EWAS for any of the four lipid traits ( $N=912$ UMRs). A Bonferroni $q<$ 0.05 cutoff was applied for significance.

Differential expression analyses. Peripheral blood mononuclear cells were purified from buffy coats originating from $450 \mathrm{ml}$ blood of healthy blood donors (Uppsala Blood Transfusion Center, Uppsala University Hospital, Sweden), using Ficoll-Paque (GE Healthcare) density-gradient centrifugation. B cells, T cells and monocytes were isolated from dedicated batches of peripheral blood mononuclear cells, using positive selection with $\mathrm{CD} 19+, \mathrm{CD} 3+$, and CD14+ beads (Miltenyi Biotec), respectively, according to the manufacturer's instructions.

RNA isolations were performed using miRNeasy Mini Kit (Qiagen). RNA library preparations were carried out on 500 ng of RNA with RNA integrity number $(\mathrm{RIN})>7$ isolated from adipocyte cells extracted from $\mathrm{AT}^{55,56}$ and blood cells (CD19+, CD3+, and CD14+) using the Illumina TruSeq Stranded Total RNA Sample preparation kit, according to manufacturer's protocol. Final libraries were analysed on a Bioanalyzer and sequenced on the Illumina HiSeq2000 (pairended 100 bp sequences). Raw reads were trimmed for quality (phred33 $\geq 30$ ) and length $(N \geq 32)$, and Illumina adapters were clipped off using Trimmomatic v. $0.32^{57}$. Filtered reads were aligned to the hg19 human reference using STAR v.2.5.1 $\mathrm{b}^{58}$. Raw read counts of UCSC genes were obtained using htseq-count v.0.6.1 (http://www-huber.embl.de/users/anders/HTSeq).

Differential expression analysis was done using DESeq2 v.1.18.159 on RNA-seq data from adipocytes isolated from adipose tissue (subcutaneous and visceral) of 20 obese individuals undergoing bariatric surgery (IUCPQ) and different blood cell types ( $N=11$ B cells; $N=20 \mathrm{~T}$ cells; $N=20$ monocytes) of healthy European individuals (Uppsala Blood Transfusion Center, Uppsala University Hospital, Sweden). We used stringent cutoffs to define adipocyte-specific expressionrequiring $\log 2$-fold-change $>2$ and $p<0.05$ across all six comparisons of adipocytes to blood cell types.

Linking gene expression to methylation in MuTHER cohort. We expanded on a previously published methylation-expression association analysis performed within the MuTHER cohort ${ }^{11}$ to assess possible long-range interactions $(+/-1 \mathrm{Mb})$ for CpGs mapping to both LMRs and UMRs. We restricted to $145,913450 \mathrm{~K} \mathrm{CpGs}$ residing in 27,258 adipose regulatory regions and tested for association to 20,326 expression probes (IlluminaHT12) for 602 individuals with matched samples. We used a similar linear mixed-effects model as described previously ${ }^{11}$, implemented with the lme 4 package ${ }^{60} \operatorname{lmer}()$ function fitted by maximum likelihood. As before, the linear mixed-effects model was adjusted for both fixed effects (age, beadchip, BS conversion efficiency, BS-treated DNA input) and random effects (family relationship and zygosity) but here we added BMI as an additional covariate. We used a likelihood ratio test to assess the significance of the gene expression effect. The $p$ value of the gene expression effect in each model was calculated from the Chisquare distribution with 1 degree of freedom (df) and $-2 \log$ (likelihood ratio) as the test statistic. In total, we tested 4,245,804 methylation to gene expression associations and assessed the false-discovery rate (FDR 10\%) using the R/Bioconductor $q$-value package ${ }^{52}$.

\section{Association of gene expression to lipids in MuTHER cohort. Associations} between gene expression levels (IlluminaHT12) and lipid status within the MuTHER cohort were modeled using a linear mixed effects model as described previously ${ }^{61}$. Briefly, the lmer function in the lme 4 package ${ }^{60}$, was fitted by maximum-likelihood. The linear mixed effects model was adjusted for age and experimental batch (fixed effects) and family relationship (twin-pairing) and zygosity (random effects). A likelihood ratio test was used to assess the significance of the phenotype effect. The $p$-value of the phenotype effect in each model was calculated from the Chi-square distribution with 1 degree of freedom using $-2 \log$ (likelihood ratio) as the test statistic.

Gene enrichment pathway analyses. Core expression analyses were performed using default settings in the Ingenuity Pathway Analysis software. Only the top 5 canonical pathways are reported. We ran the software on (1) 30 genes showing associations to both replicated adipose lipid-CpGs mapping with adipose regulatory regions and to the same lipid trait independently (see "Functional annotation of lipid-CpGs"), and (2) 52 genes directly overlapping the 68 lipid-linked adipose regulatory regions validated in whole-blood (see "Tissue-specificity of lipid-linked regulatory regions").

Conditional modeling of HDL-EWAS on SNPs. Genotypes for the 21 SNPs in the region of interest within GALNT2 (Supplementary Data 8) were generated for 148 adipose IUCPQ samples. For 56/113 discovery samples profiled via MCC-Seq using MetV1, genotypes were typed on the high-density genotyping using the Illumina HumanOmni2.5-8 (Omni2.5) BeadChip according to protocols recommended by Illumina. For 92 discovery samples profiled via MCC-Seq using MetV2, genotypes were inferred using the Bis-SNP software ${ }^{62}$, a bisulfite-sequencing variant caller, with default parameters: “-T BisulfiteGenotyper -stand_call_conf 20 -stand_emit_conf 0 -mmq $30-\mathrm{mbq} 17$-minConv 0" and with dbSNP 137 as prior SNP information. The aligned bam files were used as input file and the hg19 was used as the reference genome.
Conditional modeling of HDL-association at chr1:230313001 for the 21 SNPs in the region of interest within GALNT2 (Supplementary Data 8) was carried out independently for each SNP by adding the genotype status as a covariate in the GLMs as described in "Epigenome-wide association of plasma lipid levels" above.

Reporting summary. Further information on experimental design is available in the Nature Research Reporting Summary linked to this article.

\section{Data availability}

The methylation and expression data from the MuTHER cohort have been deposited in the ArrayExpress, https://www.ebi.ac.uk/arrayexpress/ (accession no. "E-MTAB-1866" and "E-TABM-1140". Lipid-EWAS results from the adipose and whole-blood IUCPQ cohorts as well as the whole-blood CARTaGENE cohort can be visualized in the UCSC Genome Browser by adding the following URL to "My Hubs": https://emc.genome. mcgill.ca/myHub/hub_adipose.txt. Raw MCC-Seq reads from the IUCPQ cohorts are deposited to the European Genome-phenome Archive (EGA) and available (accession no. EGAS00001003415) after approval by the Data Access Committee (DAC) designated to the study (https://www.ebi.ac.uk/ega/home). All other relevant data supporting the key findings of this study are available within the article and its Supplementary Information files or from the corresponding author upon reasonable request. A reporting summary for this Article is available as a Supplementary Information file.

Received: 14 February 2018 Accepted: 25 February 2019 Published online: 14 March 2019

\section{References}

1. Kilpinen, H. \& Dermitzakis, E. T. Genetic and epigenetic contribution to complex traits. Hum. Mol. Genet. 21, R24-R28 (2012).

2. Barres, R. \& Zierath, J. R. DNA methylation in metabolic disorders. Am. Clin. Nutr. 93, 897S-900S (2011).

3. Gluckman, P. D., Hanson, M. A., Buklijas, T., Low, F. M. \& Beedle, A. S. Epigenetic mechanisms that underpin metabolic and cardiovascular diseases. Nat. Rev. Endocrinol. 5, 401-408 (2009).

4. Elder, S. J. et al. Genetic and environmental influences on factors associated with cardiovascular disease and the metabolic syndrome. J. Lipid Res. 50, 1917-1926 (2009)

5. Yang, J. et al. Genetic variance estimation with imputed variants finds negligible missing heritability for human height and body mass index. Nat. Genet. 47, 1114-1120 (2015).

6. Dayeh, T. et al. Genome-wide DNA methylation analysis of human pancreatic islets from type 2 diabetic and non-diabetic donors identifies candidate genes that influence insulin secretion. PLoS Genet. 10, e1004160 (2014).

7. Dick, K. J. et al. DNA methylation and body-mass index: a genome-wide analysis. The Lancet 383, 1990-1998 (2014).

8. Mathers, J. C., Strathdee, G. \& Relton, C. L. in Advances in Genetics (eds Herceg, Z. \& Ushijima, T.) 1-39 (Academic Press, San Diego, CA, USA 2010).

9. Breitling, L. P., Yang, R., Korn, B., Burwinkel, B. \& Brenner, H. Tobaccosmoking-related differential DNA methylation: $27 \mathrm{~K}$ discovery and replication. Am. J. Hum. Genet. 88, 450-457 (2011).

10. Wagner, J. R. et al. The relationship between DNA methylation, genetic and expression inter-individual variation in untransformed human fibroblasts. Genome Biol. 15, R37 (2014).

11. Grundberg, E. et al. Global analysis of DNA methylation variation in adipose tissue from twins reveals links to disease-associated variants in distal regulatory elements. Am. J. Human. Genet. 93, 876-890 (2013).

12. Eichler, E. E. et al. Missing heritability and strategies for finding the underlying causes of complex disease. Nat. Rev. Genet. 11, 446-450 (2010).

13. Wildman, R. P. et al. The obese without cardiometabolic risk factor clustering and the normal weight with cardiometabolic risk factor clustering: prevalence and correlates of 2 phenotypes among the US population (NHANES 19992004). Arch. Intern. Med. 168, 1617-1624 (2008).

14. Dekkers, K. F. et al. Blood lipids influence DNA methylation in circulating cells. Genome Biol. 17, 138 (2016).

15. Pfeifferm, L. et al. DNA methylation of lipid-related genes affects blood lipid levels. Circulation 114, 000804 (2015).

16. Irvin, M. R. et al. Epigenome-wide association study of fasting blood lipids in the genetics of lipid lowering drugs and diet network study. Circulation 114, 009158 (2014)

17. Braun, K. V. et al. Epigenome-wide association study (EWAS) on lipids: the Rotterdam Study. Clin. Epigenetics 9, 15 (2017).

18. Hedman, $\AA$. K. et al. Epigenetic patterns in blood associated with lipid traits predict incident coronary heart disease events and are enriched for results 
from genome-wide association studies. Circulation 10, e001487 (2017).

19. Grundberg, E. et al. Mapping cis- and trans-regulatory effects across multiple tissues in twins. Nat. Genet. 44, 1084-1089, https://doi.org/10.1038/ng.2394 (2012).

20. Allum, F. et al. Characterization of functional methylomes by next-generation capture sequencing identifies novel disease-associated variants. Nat. Commun. 6, 7211 (2015).

21. Cheung, W. A. et al. Functional variation in allelic methylomes underscores a strong genetic contribution and reveals novel epigenetic alterations in the human epigenome. Genome Biol. 18, 50 (2017).

22. van Iterson, M., van Zwet, E. W. \& Heijmans, B. T. Controlling bias and inflation in epigenome-and transcriptome-wide association studies using the empirical null distribution. Genome Biol. 18, 19 (2017).

23. Busche, S. et al. Population whole-genome bisulfite sequencing across two tissues highlights the environment as the principal source of human methylome variation. Genome Biol. 16, 290 (2015).

24. Richard, A. J. \& Stephens, J. M. The role of JAK-STAT signaling in adipose tissue function. Biochim. Et. Biophys. Acta 1842, 431-439 (2014).

25. Zhao, P. \& Stephens, J. M. Identification of STAT target genes in adipocytes. Jak-Stat 2, e23092 (2013)

26. Stephens, J. M., Morrison, R. F., Wu, Z. \& Farmer, S. R. PPAR $\gamma$ liganddependent induction of STAT1, STAT5A, and STAT5B during adipogenesis. Biochem. Biophys. Res. Commun. 262, 216-222 (1999).

27. Kaltenecker, D. et al. Adipocyte STAT5 deficiency promotes adiposity and impairs lipid mobilisation in mice. Diabetologia 60, 296-305 (2017).

28. Priceman, S. J. et al. Regulation of adipose tissue T cell subsets by Stat 3 is crucial for diet-induced obesity and insulin resistance. Proc. Natl Acad. Sci. 110, 13079-13084 (2013).

29. Miura, S. et al. Nuclear factor 1 regulates adipose tissue-specific expression in the mouse GLUT4 gene. Biochem. Biophys. Res. Commun. 325, 812-818 (2004).

30. Kadowaki, T. et al. in Cold Spring Harbor Symposia on Quantitative Biology. 257-265 (Cold Spring Harbor Laboratory Press).

31. Hou, X. et al. CDK6 inhibits white to beige fat transition by suppressing RUNX1. Nat. Commun. 9, 1023 (2018).

32. Lu, F. \& Liu, Q. Validation of RUNX1 as a potential target for treating circadian clock-induced obesity through preventing migration of group 3 innate lymphoid cells into intestine. Med. Hypotheses 113, 98-101 (2018)

33. Bell, J. T. et al. DNA methylation patterns associate with genetic and gene expression variation in HapMap cell lines. Genome Biol. 12, R10 (2011).

34. Willer, C. J. et al. Discovery and refinement of loci associated with lipid levels. Nat. Genet. 45, 1274 (2013).

35. Smith, N. L. et al. Association of genome-wide variation with the risk of incident heart failure in adults of European and African ancestry: a prospective meta-analysis from the cohorts for heart and aging research in genomic epidemiology (CHARGE) consortium. Circulation 3, 256-266 (2010).

36. Palmer, N. D. et al. Genetic variants associated with quantitative glucose homeostasis traits translate to type 2 diabetes in Mexican Americans: the GUARDIAN (Genetics Underlying Diabetes in Hispanics) Consortium. Diabetes 64, 1853-1866 (2015)

37. Divers, J. et al. Genome-wide association study of coronary artery calcified atherosclerotic plaque in African Americans with type 2 diabetes. BMC Genet. 18, 105 (2017).

38. Chen, L. et al. Genetic drivers of epigenetic and transcriptional variation in human immune cells. Cell 167, 1398-1414. e1324 (2016)

39. Kristiansson, K. et al. Genome-wide screen for metabolic syndrome susceptibility loci reveals strong lipid gene contribution but no evidence for common genetic basis for clustering of metabolic syndrome traits. Circulation 5, 242-249 (2012)

40. Li, Q. et al. Association of the GALNT2 gene polymorphisms and several environmental factors with serum lipid levels in the Mulao and Han populations. Lipids Health Dis. 10, 160 (2011).

41. Teslovich, T. M. et al. Biological, clinical and population relevance of 95 loci for blood lipids. Nature 466, 707 (2010).

42. Marucci, A. et al. GALNT2 expression is reduced in patients with Type 2 diabetes: possible role of hyperglycemia. PLoS ONE 8, e70159 (2013).

43. Newton-Cheh, C. et al. Genome-wide association study identifies eight loci associated with blood pressure. Nat. Genet. 41, 666 (2009).

44. Demerath, E. W. et al. Epigenome-wide association study (EWAS) of BMI, BMI change and waist circumference in African American adults identifies multiple replicated loci. Hum. Mol. Genet. 24, 4464-4479 (2015).

45. Eisenstein, A. \& Ravid, K. G protein-coupled receptors and adipogenesis: a focus on adenosine receptors. J. Cell. Physiol. 229, 414-421 (2014).

46. Liu, L. \& Clipstone, N. A. Prostaglandin F2 $\alpha$ inhibits adipocyte differentiation via a Gaq-calcium-calcineurin-dependent signaling pathway. J. Cell. Biochem. 100, 161-173 (2007).
47. Marceau, P. et al. Biliopancreatic diversion with duodenal switch. World J. Surg. 22, 947-954 (1998).

48. Vohl, M. C. et al. A survey of genes differentially expressed in subcutaneous and visceral adipose tissue in men. Obes. Res. 12, 1217-1222 (2004).

49. Warren, H. R. et al. Genome-wide association analysis identifies novel blood pressure loci and offers biological insights into cardiovascular risk. Nat. Genet. 49, 403 (2017).

50. Li, H. \& Durbin, R. Fast and accurate short read alignment with Burrows-Wheeler transform. Bioinformatics 25, 1754-1760 (2009).

51. Johnson, M. D., Mueller, M., Game, L. \& Aitman, T. J. Single nucleotide analysis of cytosine methylation by whole-genome shotgun bisulfite sequencing. Curr. Protoc. Mol. Biol. 21, 21-28 (2012).

52. Storey, J. D. \& Tibshirani, R. Statistical significance for genomewide studies. Proc. Natl Acad. Sci. 100, 9440-9445 (2003).

53. Wickham, H. ggplot2: Elegant Graphics for Data Analysis. (Springer, New York, 2016).

54. Heinz, S. et al. Simple combinations of lineage-determining transcription factors prime cis-regulatory elements required for macrophage and B cell identities. Mol. Cell 38, 576-589 (2010).

55. Guenard, F. et al. Association of LIPA gene polymorphisms with obesityrelated metabolic complications among severely obese patients. Obesity 20 2075-2082 (2012)

56. Turcot, V. et al. LINE-1 methylation in visceral adipose tissue of severely obese individuals is associated with metabolic syndrome status and related phenotypes. Clin. Epigenetics 4, 10 (2012).

57. Lohse, M. et al. RobiNA: a user-friendly, integrated software solution for RNA-Seq-based transcriptomics. Nucleic Acids Res. 40, gks540 (2012).

58. Dobin, A. et al. STAR: ultrafast universal RNA-seq aligner. Bioinformatics 29, 15-21 (2013).

59. Love, M. I., Huber, W. \& Anders, S. Moderated estimation of fold change and dispersion for RNA-seq data with DESeq2. Genome Biol. 15, 550 (2014).

60. Bates, D., Mächler, M., Bolker, B. \& Walker, S. Fitting linear mixed-effects models using lme4. arXiv preprint arXiv:1406.5823 (2014).

61. Small, K. S. et al. Identification of an imprinted master trans regulator at the KLF14 locus related to multiple metabolic phenotypes. Nat. Genet. 43, 561 (2011).

62. Liu, Y., Siegmund, K. D., Laird, P. W. \& Berman, B. P. Bis-SNP: combined DNA methylation and SNP calling for bisulfite-seq data. Genome Biol. 13, R61 (2012).

\section{Acknowledgements}

This work was supported by a Canadian Institute of Health Research (CIHR) team gran awarded to E.G. and M.L. (TEC-128093), a CIHR Foundation grant awarded to E.G (148391) and the CIHR funded Epigenome Mapping Center at McGill University (EP1120608) awarded to T.P. and M.L. E.G. holds the Roberta D. Harding \& William F. Bradley, Jr. Endowed Chair in Genomic Research and T.P. holds the Dee Lyons/Missouri Endowed Chair in Pediatric Genomic Medicine. A.T. is the director of a Research Chair in Bariatric and Metabolic Surgery. M.C.V. holds the Canada Research Chair in Genomics Applied to Nutrition and Health (Tier 1). F.A. held a studentship from The Fonds de recherche du Québec (FRSQ) during part of this study. The study was further supported by the Swedish Rheumatism Association and King Gustaf V's 80-years Foundation together with The Swedish Research Council and Wallenberg Foundation awarded to L.R. This study was also supported by the NIHR Oxford Biomedical Research Center. The views expressed are those of the authors and not necessarily those of the NHS, the NIHR or the Department of Health. The MuTHER Study was funded by a program grant from the Wellcome Trust $(081917 / \mathrm{Z} / 07 / \mathrm{Z})$ and core funding for the Wellcome Trust Center for Human Genetics (090532). The TwinsUK study was funded by the Wellcome Trust and European Community's Seventh Framework Program (FP7/ 2007-2013). The TwinsUK study also receives support from the National Institute for Health Research (NIHR)-funded BioResource, Clinical Research Facility and Biomedical Research Center based at Guy's and St Thomas' NHS Foundation Trust in partnership with King's College London. We thank the NIH Roadmap Epigenomics Consortium and the Mapping Centers (http://nihroadmap.nih.gov/epigenomics/) for the production of publicly available reference epigenomes. Specifically, we thank the mapping centers at MGH/BROAD and UCSF for generation of human adipose (donor 92 and 7) and peripheral blood (TC014 and TC015) reference epigenomes used in this study, respectively. We further thank additional members of the MuTHER consortium for providing valuable data for this study. Please see the Supplementary Note in the Supplementary Information document for a full list of additional MuTHER members not already included in the author list.

\section{Author contributions}

E.G. conceived the study. E.G., T.P., M.C.V., A.T., and M.L. designed experiments. F.G, L.R., A.T., and M.C.V. collected, prepared and/or provided the clinical samples. E.G. and F.A. lead data analyses. F.A., M.M.S., and E.B. performed experiments. F.A., A.K.H., W. A.C., X.S., and J.V. analyzed data. T.K. and B.G. provided bioinformatics support. A.K. 
H., M.I.M., S.B., C.M., and P.D. provided replication data. F.A. generated figures with contributions from W.A.C. F.A. and E.G. drafted the manuscript. All authors reviewed and contributed feedback on the final manuscript.

\section{Additional information}

Supplementary Information accompanies this paper at https://doi.org/10.1038/s41467019-09184-z.

Competing interests: A.T. receives research funding from Johnson \& Johnson Medical Companies and Medtronic for studies unrelated to this manuscript. The remaining authors declare no competing interests.

Reprints and permission information is available online at http://npg.nature.com/ reprintsandpermissions/

Journal peer review information: Nature Communications thanks the anonymous reviewers for their contribution to the peer review of this work. Peer reviewer reports are available.
Publisher's note: Springer Nature remains neutral with regard to jurisdictional claims in published maps and institutional affiliations.

(c) (i) Open Access This article is licensed under a Creative Commons Attribution 4.0 International License, which permits use, sharing, adaptation, distribution and reproduction in any medium or format, as long as you give appropriate credit to the original author(s) and the source, provide a link to the Creative Commons license, and indicate if changes were made. The images or other third party material in this article are included in the article's Creative Commons license, unless indicated otherwise in a credit line to the material. If material is not included in the article's Creative Commons license and your intended use is not permitted by statutory regulation or exceeds the permitted use, you will need to obtain permission directly from the copyright holder. To view a copy of this license, visit http://creativecommons.org/ licenses/by/4.0/.

(C) The Author(s) 2019 